\title{
Probing molecular absorption under slow-light propagation using a photonic crystal waveguide
}

\author{
Isabelle Dicaire, ${ }^{1}$ Alfredo De Rossi, ${ }^{2}$ Sylvain Combrié, ${ }^{2}$ and Luc Thévenaz ${ }^{1, *}$ \\ ${ }^{1}$ École Polytechnique Fédérale de Lausanne (EPFL), Institute of Electrical Engineering, \\ STI-GR-SCI-LT Station 11, Lausanne 1015, Switzerland \\ ${ }^{2}$ Thales Research and Technology, Route Départementale 128, Palaiseau Cedex 91767, France \\ *Corresponding author: luc.thevenaz@epfl.ch
}

Received August 23, 2012; revised October 17, 2012; accepted October 18, 2012; posted October 19, 2012 (Doc. ID 174897); published November 28, 2012

\begin{abstract}
High-resolution infrared absorption spectroscopy of acetylene gas is demonstrated in a dispersion-engineered photonic crystal waveguide under slow-light propagation. Experimental enhancement factors of 0.31 and 1.00 are obtained for TE and TM polarization, respectively, for group indices ranging from 1.5 to 6.7. The dependence of molecular absorption on the evanescent electric-field distribution and on the group index under structural slowlight illumination is experimentally demonstrated and confirmed by time-domain simulations. @ 2012 Optical Society of America

OCIS codes: $\quad 230.5298,280.4788,300.6320,260.2030$.
\end{abstract}

Photonic crystal (PhC) waveguides are attractive optical sensing tools based on the evanescent field detection principle to monitor hazardous gases and analytes in aqueous environment. They require small sample volumes and short response times due to their ultracompact dimensions. Refractive index sensing was recently demonstrated in $\mathrm{PhC}$ waveguides using $\mathrm{PhC}$ optical cavities with air slots embedded in the defect region [1-3]. While this technique allows for very sensitive measurements of refractive index changes, it cannot specifically identify the chemical species under study. In this case infrared (IR) absorption spectroscopy is a powerful and highly selective technique that can identify molecular species from their individual spectroscopic fingerprint. The BeerLambert law describes the intensity of a light signal decaying through an absorbing medium:

$$
I=I_{0} \exp (-\gamma \alpha L)
$$

where $I_{0}$ is incident light intensity, $\alpha$ is the highly selective wavelength-dependent absorption coefficient, $L$ is the optical path length, and $\gamma$ is the absorption enhancement factor equal to 1 for free beam interaction. Since the optical path length is notably small in PhC waveguides, it was previously suggested to use slow-light propagation to locally enhance light-matter interactions, thus enhancing the absorption sensitivity [4]. Gas sensing measurements of carbon dioxide gas and methane gas were recently performed in dispersion-engineered bulk $\mathrm{PhCs}$ and $\mathrm{PhC}$ waveguides, respectively [5,6]. Experimental enhancement factors $\gamma$ of 2-3 were reported in [5] for group indices of $c / v_{g} \sim 2$, and a high absorption sensitivity was reported in [6] - however without any comparison with free-space absorption.

In this Letter, high-resolution IR absorption spectroscopy of acetylene gas is demonstrated in dispersionengineered GaInP $\mathrm{PhC}$ waveguides under slow-light propagation. Individual absorption profiles are obtained for both TE and TM polarizations to highlight the various factors contributing to the absorption coefficient, namely its critical dependence on the group index and the electric-field intensity. Experimental enhancement factors are obtained by measuring the corresponding absorption coefficients under free-space propagation for exactly the same path length. The gas sensing measurements are confirmed by numerical simulations of molecular absorption via the evanescent field of $\mathrm{PhC}$ waveguides in the presence of wavelength-dependent dispersion.

The $\mathrm{PhC}$ waveguide samples consist of a $180 \mathrm{~nm}$ thick GaInP slab having a triangular lattice of air holes with a lattice constant of $486 \mathrm{~nm}$. They were fabricated via inductively coupled plasma etching followed by chemical wet etching in order to obtain a self-standing membrane [7]. To modify their structural dispersion properties, the size of the first row of holes was increased from $0.21 a$ to $0.24 a$ in sample 1 and from $0.23 a$ to $0.26 a$ in sample 2 . The $1.5 \mathrm{~mm}$ long waveguides include mode adapters on both end facets to gradually build the slow-light mode and to reduce Fabry-Perot oscillations due to the strong impedance mismatch between incident modes (freespace or optical-fiber modes) and waveguide modes [].

Molecular spectroscopy was performed in PhC waveguides using the following experimental procedure. The optical coupling setup comprising two micropositioning stages and a moving sample holder was placed inside a gas chamber having optical feedthrough for remote monitoring. Lensed optical fibers having a working distance of $14 \mu \mathrm{m}$ provided optical coupling to the $\mathrm{PhC}$ waveguides while making fully negligible the absorption of incident and transmitted light by gas molecules in the fiber-PhC gaps. An optical imaging setup consisting of a microscope objective, an illumination system, and an IR-sensitive camera was used to ensure accurate optical coupling between the lensed fibers and $\mathrm{PhC}$ waveguides. The polarization state incident on the samples was set to TE and TM independently using a linear polarizer providing a fixed arbitrary polarization followed by a polarization controller to adjust the polarization state. TE polarization was set by observing a cutoff in the transmission spectrum of a PhC sample using an optical spectrum 

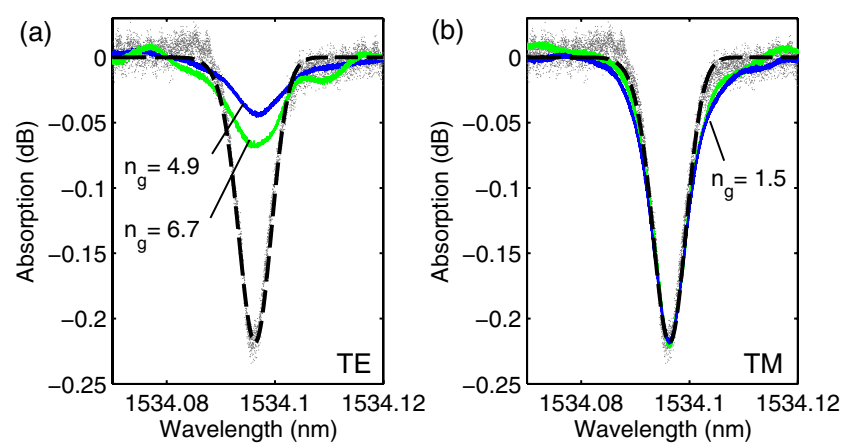

Fig. 1. (Color online) (a) Acetylene P15 absorption line for the TE polarization for $n_{g}=4.9$ and $n_{g}=6.7$. Experimental free-space absorption (dotted profile) and its Gaussian fit (dashed curve) are also shown. (b) Same for TM polarization, $n_{g}=1.5$ for the two PhC samples. Lineshape details can be found in Table 1 .

analyzer (OSA) and a broadband source emitting in the wavelength range $1520-1570 \mathrm{~nm}$. TM polarization was set by observing no spectral cutoff on the OSA, indicating that light no longer experienced multiple reflections by the periodic PhC structure.

The gas chamber was then hermetically closed and air was evacuated using a vacuum pump without causing any significant change in transmitted intensity $( \pm 1 \mathrm{~dB})$. The gas chamber was filled with 50 Torr (67 mbars) of acetylene gas; this value was chosen to minimize the absorption linewidth while keeping a full peak absorption. To obtain high-resolution absorption profiles the injection current of a distributed-feedback (DFB) laser diode operating at $1534.1 \mathrm{~nm}$ was modulated by a ramp signal to sweep the DFB laser line across the P15 acetylene absorption line. Group indices were measured using an optical frequency domain interferometric technique comprising a tunable laser source and a Mach-Zehnder interferometer with the $\mathrm{PhC}$ waveguide sample placed in one arm and followed by digital filtering [7].

The experimental absorption lines are presented in Fig. 1 for both TE and TM polarizations (blue and green solid curves, color online) and for two different waveguides with different $n_{g}$ values. The dashed curve represents absorption under free-space propagation for exactly the same path length as the PhC samples $(L=1.5 \mathrm{~mm})$.

As illustrated in Fig. 1 the experimental absorption efficiency is greater for the TM polarization with $n_{g}=1.5$ than for the TE one for which $n_{g}=[4.9 ; 6.7]$. This is a very surprising result considering the expected enhancement of molecular absorption due to slow-light propagation that occurs only for the TE mode. This result is due to the fact that most of the electric field is located inside the high-index membrane for the TE mode while for TM mode the electric field is enhanced in the lower-index material. Boundary conditions at material interfaces state that for an electric field perpendicular to the highindex dielectric slab (i.e., TM pol.), the electric displacement field $D=\epsilon E$ must satisfy continuity at its interface, resulting in a relative evanescent electric field enhancement of $\epsilon_{\text {slab }} / \epsilon_{0}$ for TM polarization and a continuous weak evanescent field for TE polarization.

To circumvent low TE electric-field intensities, a narrow air slot can be added in the defect region of a $\mathrm{PhC}$ waveguide, thereby causing a large field discontinuity for $\mathrm{TE}$ polarization and electric-field enhancement in the air slot [9], leading to enhanced optical interactions [10]. This can also be done through the use of slow- light propagation in dispersive structures as demonstrated in [11] for fiber ring cavities and shown hereby for $\mathrm{PhC}$ devices.

To experimentally compare the dependence of molecular absorption on the group index, several conditions must be satisfied. One intuitive requirement is that the absorption linewidths should be equal since the peak absorption is a function of the absorption linewidth for any Lorentzian or Gaussian profile. The electric-field intensity $\|E\|^{2}$ located within the lower-index material should also be identical for all measurements. Table 1 presents the experimental peak absorption values, absorption linewidths, and equivalent mode field radii $w$ calculated from the transit-time contribution to line broadening $\Delta \nu_{\mathrm{tt}}$ due to the short transit time of molecules through the electric field, which limits the duration of the electricdipole interaction:

$$
\Delta \nu_{\mathrm{tt}}(\mathrm{FWHM})=C \frac{u}{\pi w},
$$

where $C=\sqrt{2 \ln 2}$ for free-space Gaussian beams and $C=1$ for evanescent fields and where $u=432.55 \mathrm{~m} / \mathrm{s}$ is the most probable velocity of acetylene molecules. Since the TE linewidths and field radii are equal, the peak absorption values can be directly compared to group indices. We find that the absorption coefficient is approximately linearly proportional to $n_{g}$ when all other relevant quantities are equal.

To confirm this linear dependence, time-domain simulations of molecular absorption were realized using our own implementation of a finite-difference timedomain algorithm with appropriate boundary conditions $[E(x+a)=E(x) \exp (i k a)$, with $a$ the lattice constant and $k$ the wavevector]. The simulated electromagnetic (EM) field distributions and dispersion relation were then used to calculate enhancement factors.

Starting from Eq. (1), the Poynting theorem is applied to relate the flux of the EM energy, through two parallel surfaces $S$ transverse to the waveguide axis and spaced

Table 1. Experimental and Simulated Absorption Profile Characteristics

\begin{tabular}{lccccccc}
\hline $\begin{array}{l}\text { Polarization } \\
\text { (or propagation) }\end{array}$ & $n_{g}$ & $\begin{array}{c}\text { Linewidth } \\
{[\mathrm{GHz}]}\end{array}$ & $\begin{array}{c}\text { Exper. Field } \\
\text { Radius }[\mu \mathrm{m}]\end{array}$ & $\begin{array}{c}\text { Exper. Abs. } \\
{[\mathrm{dB}]}\end{array}$ & $\begin{array}{c}\text { Exper. } \\
\text { Enhanc. }\end{array}$ & $\begin{array}{c}\text { Simul. } \\
\text { Enhanc. }\end{array}$ & $\begin{array}{c}\text { Simul. Evanes. Field } \\
\text { Dimensions [ } \mu \mathrm{m}]\end{array}$ \\
\hline Free-space & 1.0 & $0.87 \pm 0.05$ & $6.00 \pm 2.00$ & $0.218 \pm 0.020$ & - & - & - \\
TM & 1.5 & $0.94 \pm 0.01$ & $1.20 \pm 0.20$ & $0.219 \pm 0.003$ & $1.00 \pm 0.05$ & 1.12 & {$[0.47 \pm 0.03] \times[1.70 \pm 0.07]$} \\
TE & 4.9 & $1.40 \pm 0.10$ & $0.21 \pm 0.04$ & $0.044 \pm 0.001$ & $0.20 \pm 0.01$ & 0.19 & {$[0.13 \pm 0.01] \times[0.27 \pm 0.06]$} \\
TE & 6.7 & $1.50 \pm 0.30$ & $0.19 \pm 0.13$ & $0.068 \pm 0.001$ & $0.31 \pm 0.02$ & 0.25 & {$[0.13 \pm 0.01] \times[0.27 \pm 0.06]$} \\
\hline
\end{tabular}




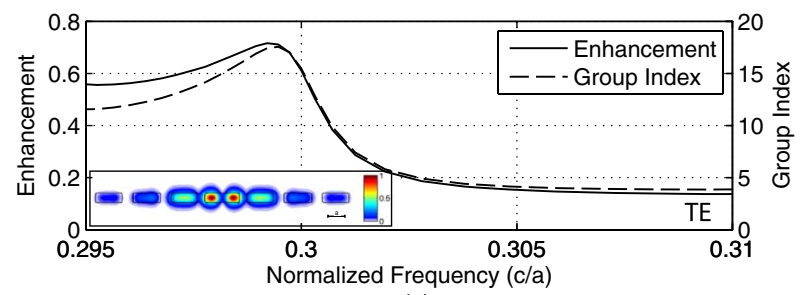

(a)

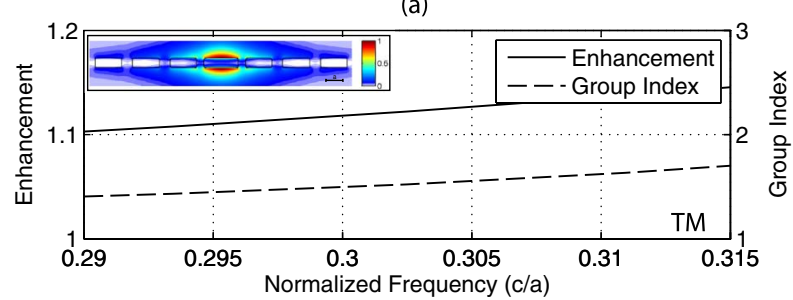

(b)

Fig. 2. (Color online) (a) Simulated enhancement factors (solid line) and group indices (dashed line) as a function of the normalized frequency for the TE polarization with the electric-field intensity distribution shown in the inset. (b) Same for the TM polarization.

by $d x$, to the absorption of the energy inside the enclosed volume $V$. We note that the group velocity is the ratio of the spatial average of the Poynting vector to the EM energy [12] thus $I d x=0.5 \operatorname{Re}\left(\int_{S} \mathbf{E} \times \mathbf{H}^{*} \mathrm{~d} S\right) \mathrm{d} x=0.5 \epsilon_{0} v_{g}$ $\int_{V} \operatorname{Re}\left(\mathbf{E} \epsilon_{\mathbf{r}} \mathbf{E}^{*}\right) \mathrm{d} V$, where $\epsilon_{r}$ is the dielectric permittivity. This yields the formula below:

$$
\alpha=-\frac{d I}{I d x}=\frac{\omega \int_{V} \operatorname{Im}\left(\mathbf{E}_{\mathbf{r}} \mathbf{E}^{*}\right) \mathrm{d} V}{v_{g} \int_{V} \operatorname{Re}\left(\mathbf{E} \epsilon_{\mathbf{r}} \mathbf{E}^{*}\right) \mathrm{d} V} \approx \frac{\operatorname{Im}\left(\epsilon_{r}\right) \omega f}{v_{g}} .
$$

Here $f$ is the fraction of the electric field in the area where the absorption takes place. Assuming the absorption is nonzero and uniform outside the $\mathrm{PhC}$, this leads to a simple expression $f=\int_{V_{a}}|E|^{2} \mathrm{~d} V / \int_{V} \operatorname{Re}\left(\epsilon_{r}\right)|E|^{2} \mathrm{~d} V$ representing the (weighted) overlap of the spatial distribution of the optical mode with the volume $V_{a}$ outside the PhC. Thus Eq. (3) implies that absorption depends on the group index and on the spatial distribution of the field, which are both dispersive.

Figure 2 presents the theoretical absorption coefficient normalized by that obtained in free-space propagation for both TE and TM polarizations and with the distribution of the electric-field intensity shown in the insets. As expected, the absorption coefficient is strongly dependent on the electric-field intensity outside the PhC slab leading to larger enhancement factors for the TM mode. In addition the simulated enhancement factors retrieved from Fig. $\underline{2}$ for several group-index values are presented in Table $\underline{1}$ and agree relatively well with the gas sensing measurements; small discrepancies seen for the $n_{g}=6.7$ values are believed to be due to differences in electricfield distributions. Finally, the evanescent mode field radii calculated from the experimental absorption linewidths show good agreement with the simulated electric field distributions displayed in the insets of Fig. 2 for both polarizations.

In summary, we have demonstrated high-resolution IR absorption spectroscopy of acetylene gas in dispersionengineered $\mathrm{PhC}$ waveguides having group indices ranging from 1.5 to 6.7. Experimental enhancement factors were confirmed by modeling molecular absorption as a function of the evanescent electric-field intensity and the group index under structural slow-light illumination. These results indicate that the distribution of the electricfield intensity is fundamental in gas sensing measurements and must not be confused with the optical intensity distribution; they are substantially different quantities in $\mathrm{PhC}$ waveguides. Practically, it turns out that enhancing the electric-field intensity overlapping the absorbers impacts more decisively on the absorption enhancement than searching for extreme group-index rising.

This work is funded by the European Space Agency through Grant No. 20200/06/NL/PA and by the Swiss National Office for Education and Research through Project COST C06.0015 and SNF 20020-121860.

\section{References}

1. A. Di Falco, L. O'Faolain, and T. F. Krauss, Appl. Phys. Lett. 94, 063503 (2009).

2. J. Jágerská, H. Zhang, Z. Diao, N. Le Thomas, and R. Houdré, Opt. Lett.35, 2523 (2010).

3. B. Wang, M. A. Dündar, R. Nötzel, F. Karouta, S. He, and R. W. van der Heijden, Appl. Phys. Lett. 97, 151105 (2010).

4. N. A. Mortensen and S. Xiao, Appl. Phys. Lett. 90, 141108 (2007).

5. D. Pergande, T. M. Geppert, A. von Rhein, S. L. Schweizer, R. B. Wehrspohn, S. Moretton, and A. Lambrecht, J. Appl. Phys. 109, 083117 (2011).

6. W.-C. Lai, S. Chakravarty, X. Wang, C. Lin, and R. T. Chen, Opt. Lett. 36, 984 (2011).

7. P. Colman, S. Combrié, G. Lehoucq, and A. De Rossi, Opt. Express 20, 13108 (2012).

8. Q. V. Tran, S. Combrié, P. Colman, and A. De Rossi, Appl. Phys. Lett. 95, 061105 (2009).

9. Q. Xu Almeida, C. A. Barrios, and M. Lipson, Opt. Lett. 29, 1209 (2004).

10. R. W. Boyd, J. Opt. Soc. Am. B 28, A38 (2011).

11. L. Thévenaz, I. Dicaire, and S. Chin, Proc. SPIE 8273, 82731D (2012)

12. P. Yeh, J. Opt. Soc. Am. 69, 742 (1979). 